\title{
Uso de encuestas preliminares en la plataforma educativa para la creación de perfiles de estudiantes en las primeras semanas de docencia
}

\author{
Gemma Minero Alejandre \\ Profesora ayudante doctora de Derecho Civil de la \\ Universidad Autónoma de Madrid \\ gemma.minero@uam.es
}

\section{Extracto}

\begin{abstract}
El escaso número de horas de docencia en los grados universitarios conduce al profesorado a situaciones de improvisación en la reorganización de programas y materiales, y genera la sensación de no conocer a su estudiantado hasta bien entrado el semestre. En este trabajo se propone la introducción generalizada de la figura de la encuesta inicial o preliminar en la docencia. La clave es la programación de la encuesta con una antelación suficiente a la fecha de inicio de la docencia y la publicidad de la existencia de dicha encuesta entre los estudiantes, así como la configuración de preguntas que realmente se ajusten a los objetivos del docente. Por tanto, la encuesta inicial ha de tener un reflejo en la programación de la docencia. Esta técnica no solo será útil en los procesos de acreditación y Docentia. Al profesorado nos servirá para ganar mayor seguridad en la configuración del cronograma y en el empleo de técnicas educativas. A los alumnos les ayudará a incrementar la consciencia sobre el esfuerzo que los docentes invierten en conocer sus expectativas de aprendizaje, así como el perfil de cada grupo, y será una buena oportunidad para implementar su papel activo dentro del proceso de aprendizaje.
\end{abstract}

Palabras clave: educación superior; calidad educativa; tecnologías de la información y la comunicación (TIC); encuesta; evaluación continua.

Cómo citar: Minero Alejandre, G. (2019). Uso de encuestas preliminares en la plataforma educativa para la creación de perfiles de estudiantes en las primeras semanas de docencia. Tecnología, Ciencia y Educación, 14, 127-147. 


\title{
Use of preliminary surveys in the educational platform for the creation of student profiles in the first weeks of teaching
}

\author{
Gemma Minero Alejandre
}

\begin{abstract}
Sometimes factors such as the -limited- number of hours of lecture in the current educational environment drive the faculty members to improvisation when re-organizing schedule and materials. This study suggests the use of an initial survey in each group. The key point when implementing this proposal implies giving the students access to the survey before the lectures start and giving enough time. The questions contained within the survey must be based in -and only in- the educational aims of the course. Finally, it is important to take into account that there must be a real interest in the educator in knowing or following the evolution of the students in relation to the questions contained within the initial survey. Therefore, the results of the initial survey should be reflected both in the schedule and materials of the course. This strategy may increase the faculty's level of confidence when creating and adapting the schedule and choosing the educative methodologies to be applied. Finally, initial surveys can contribute to build the students' conscience of the effort made by the faculty in knowing individuals and groups. In the future, it may be a good opportunity for students enrolling an active role in their learning process. Besides, surveys may be useful in education assessments processes, such as Docentia programme.
\end{abstract}

Keywords: higher education; education quality; information and communication technologies (ICT); survey; continuous assessment.

Citation: Minero Alejandre, G. (2019). Use of preliminary surveys in the educational platform for the creation of student profiles in the first weeks of teaching. Tecnologia, Ciencia y Educación, 14, 127-147. 


\section{Sumario}

1. Introducción

2. Objetivos

3. Método

4. Diseño y confección

5. Resultados y adaptaciones

6. Conclusiones

Referencias bibliográficas

Nota: este trabajo es una extensión de la ponencia ganadora del Premio al Uso Innovador de Herramientas Digitales para la Docencia, concedido en el marco de la I Jornada InnovaUDIMA con Tecnología Educativa (JIUTE), organizada por la Universidad a Distancia de Madrid, UDIMA, el 11 de marzo de 2019. 


\section{Introducción}

La rápida dinámica de los grados universitarios, que exige un número de seminarios muy reducido e impide realizar con el estudiantado todas las prácticas que nos gustaría poder llevar a cabo como docentes, nos genera la sensación de improvisación de actuaciones o, en otras palabras, de conocer a los estudiantes una vez les vemos participar en el aula y corregimos sus ejercicios. Sin embargo, nuestra docencia puede ser mucho más provechosa si hacemos ese trabajo de conocimiento del grupo y de los individuos con carácter previo al inicio de la docencia. Con esta finalidad podemos servirnos de la posibilidad que nos ofrece la plataforma educativa Moodle u otras plataformas educativas alternativas para realizar encuestas iniciales, configurando las preguntas y la propia naturaleza de la encuesta en función de cuál sea la información que queremos conocer de los estudiantes antes de empezar la impartición de la asignatura y/o en las primeras semanas de docencia. La atención a la diversidad de los estudiantes es, a día de hoy, tanto un deber como una necesidad para el personal docente, dada la heterogeneidad de perfiles presentes en los diferentes grupos y cursos, y dado también el incremento del número de itinerarios formativos previos, así como de oferta de programas de movilidad nacional e internacional. Ello debe acompañarse de medidas implementadas por la universidad para transmitir información acerca de estudiantes con necesidades especiales y datos acerca de las medidas de adaptación de la evaluación y la docencia adecuadas para cada caso, en cumplimiento del objetivo integral de consecución de una universidad inclusiva ${ }^{1}$.

Con todo, la autora de este trabajo es consciente de la necesidad de profundizar en el análisis del impacto que la herramienta que se propone tiene o puede tener a corto, medio y largo plazo, partiendo de una realidad: los estudios académicos publicados, relativos a la figura de la encuesta, no aportan datos cualitativos y/o cuantitativos sobre el impacto en la

\section{El escaso número de horas de docencia genera en el profesor la sensación de no conocer a sus estudiantes hasta bien entrado el semestre. La encuesta inicial permite al docente conocer por adelantado el perfil de los grupos de estudiantes, gracias a los datos proporcionados por ellos}

1 Dado el carácter especialmente sensible de estos datos, es importante destacar el deber que tiene el centro educativo de respetar la normativa de protección de datos personales, así como el especial cuidado que ha de tener en la recogida del consentimiento de los titulares de los datos y en la implementación de medidas específicas en su tratamiento. 
mejora docente que el empleo de esta herramienta pueda llevar consigo en la fase inicial de la docencia. En efecto, los trabajos citados en este artículo versan sobre la implementación de la encuesta en la fase final de la docencia.

Esta herramienta, en cierta medida, puede contribuir a aumentar la presencia docente percibida por los estudiantes, así como al empoderamiento de los profesores, pues estos conocerán de primera mano y desde el principio del semestre cuáles son las expectativas de aprendizaje del grupo ${ }^{2}$. Del resultado de dicha encuesta, calculado en forma de estadística por la propia plataforma educativa, podemos obtener conclusiones que mejorarán nuestra capacidad para adaptarnos a los diferentes perfiles y características de cada uno de nuestros grupos, con la consiguiente potencial repercusión positiva en las encuestas que se realicen al finalizar la asignatura y, ulteriormente, en los procesos de acreditación y evaluación docentes, como Docentia, lo que cerrará el círculo de retroalimentación del profesor (García y Rodríguez, 2018). Asimismo, es interesante destacar el papel de la herramienta que se propone en este trabajo a la hora de elaborar el autoinforme docente, una vez finalizada la asignatura en cuestión, teniendo en cuenta que, como destacan García y Rodríguez (2018), la utilidad del autoinforme es exponer nuestras condiciones de docencia y valorar la adecuación de la selección de objetivos, contenidos, metodología y sistema de evaluación de estudiantes. En efecto, el autoinforme no ha de tener otras finalidades carentes de contenido reflexivo ${ }^{3}$. Por todo ello, la herramienta que se propone estará presente en tres dimensiones fundamentales ${ }^{4}$ :

- Planificación de la docencia.

La utilidad de la encuesta inicial

- Desarrollo de la enseñanza. tiene tres dimensiones: planificación de la docencia, desarrollo de la enseñanza y resultados

- Resultados.

2 Acerca de la figura de la presencia docente, véase De Pablo (2017).

3 Sobre este punto se han pronunciado también Rodríguez (1994) y Villa (1985).

4 Debe tenerse en cuenta que, conforme a la teoría clásica de Marsh (1987), las encuestas tienen una triple utilidad:

- Para los docentes sirven de retroalimentación acerca de su desempeño profesional.

- Para los estudiantes son efectivas a la hora de realizar la selección o la matrícula de asignaturas y docentes.

- Para los sujetos que ocupan puestos de gestión son un elemento indispensable en la toma de decisiones sobre la contratación del profesorado. 
El desarrollo y la mejora de técnicas que permitan una rápida adaptación al perfil y a las expectativas de los estudiantes es crucial en el caso de los docentes de una Facultad de Derecho, dado que entre nuestra docencia se encuentra la impartición de asignaturas no solo en la propia facultad, sino también en otras facultades y grados cercanos, de carácter introductorio muchas veces -por ejemplo, Introducción al Derecho o similar-, que forman parte de los planes de estudio de carreras como las de Economía, Turismo, Administración y Dirección de Empresas, Gestión Aeronáutica, Periodismo, etc. De ahí también la heterogeneidad del estudiantado que recibe nuestra docencia y que espera que las metodologías empleadas, los contenidos impartidos y los materiales proporcionados sean acordes con la idiosincrasia de su grado y no tengan en cuenta únicamente el perfil del estudiante del grado de Derecho.

\section{La heterogeneidad de nuestro estudiantado hace especialmente adecuado el uso de la encuesta inicial para conocer sus perfiles}

El uso de encuestas iniciales no es una propuesta nueva, sino que se trata de una práctica que viene siendo empleada por el profesorado. Lo novedoso de este estudio es el hecho de tratar de pasar de una encuesta-tipo, esto es, de una encuesta que el estudiante considere genérica, a una encuesta específica, adaptada al concreto grupo de docencia a que se dirige, para intentar cumplir los objetivos que seguidamente se exponen, que no se persiguen con las encuestas de corte generalista. De ahí que se obvien preguntas genéricas o no necesarias -como puede ser el nivel de inglés de los estudiantes o la opción en la que se eligió cursar esa carrera en una universidad concreta, así como la nota que el alumno obtuvo en asignaturas de la misma rama temática- cuando las respuestas sean indiferentes para el desarrollo de la asignatura en cuestión. Por tanto, en la fase de confección de la encuesta ha de tenerse en cuenta no solo el grado concreto, sino también el curso al que va dirigida la encuesta, el carácter troncal u optativo de la asignatura, la presencia de un único docente que imparta la asignatura o de varios, así como el número de

En la encuesta inicial deben evitarse las preguntas que sean genéricas y no necesarias

\section{Objetivos}

El objetivo general de la práctica docente que se presenta es sencillo: se busca estudiar la implementación de encuestas no solo al finalizar la asignatura, sino también al comienzo de la misma, y mejorar el funcionamiento de estas encuestas iniciales, es decir, su utilidad dual para el profesorado y el estudiantado. En la confección de esta herramienta se ha empleado la plataforma educativa Moodle, pero las conclusiones de este estudio igualmente 
podrían trasladarse a otras plataformas alternativas que permitan la realización de encuestas iniciales y finales propias de cada docente, distintas de las encuestas genéricas realizadas por el centro educativo. Por el contrario, los resultados no serán extensibles a aquellas plataformas que no permitan personalizar la forma de evaluación ${ }^{5}$. Con ello se trata de conseguir el objetivo que la doctrina propugna para todo proceso de evaluación docente: producir cambios encaminados al logro de una mayor calidad universitaria con el fin último de conducir y premiar la excelencia docente ${ }^{6}$.

Si bien la autora de este trabajo únicamente ha aplicado esta herramienta a la docencia universitaria, nada obsta a que su implementación pueda extenderse a la educación primaria, a la secundaria y al bachillerato, siempre que los contenidos de las encuestas se adapten de manera efectiva a los programas y a los objetivos de cada tipo de docencia.

Se parte de la base de la facilidad de manejo de la plataforma online educativa empleada $y$, en particular, de la rapidez para configurar encuestas sencillas, cuyos contenidos pueden adaptarse a los diferentes grupos de docencia que tengamos encomendados, con independencia de su tamaño y tanto si se trata de grupos de docencia teórica de tipo magistral como si estamos ante grupos de seminarios.

El primer objetivo específico es la recopilación de aquella información relativa a los estudiantes que sea de utilidad para los docentes, algo que va de la mano del segundo objetivo específico: evitar que la obtención de esta información suponga una carga de trabajo excesiva para ambos grupos de sujetos. Solamente si los resultados de dichas encuestas pueden ser procesados con rapidez y sencillez, se podrá cumplir el tercer objetivo específico: su utilización para ajustar la idiosincrasia de las actividades e, incluso, para fijar hitos en el calendario a lo largo del semestre de impartición de la asignatura.

Pensamos que la herramienta cuyo uso proponemos en este estudio podría estar especialmente indicada para los docentes que tenemos a nuestro cargo diversos grupos y, en particular, para clases de tipo seminario. En efecto, la encuesta admite discriminar y adaptar cada pregunta al tipo de grupo, y permite comparar con mucha facilidad los resultados obtenidos

Debe evitarse que la obtención de información sobre el perfil del grupo suponga una carga de trabajo excesiva para docentes y estudiantes en función del grupo de que se trate. También se entiende que esta herramienta resulta ciertamente útil cuando se trata de tutores de trabajos fin de grado o fin de máster, aunque el número de estudiantes que le corresponda tutelar a cada docente sea inferior a la media de estudiantes matriculados en el resto de asignaturas del grado o posgrado.

5 Acerca de la utilidad de Moodle para fomentar las interacciones entre profesores y alumnos, véase Iglesias, Olmos, Torrecilla y Mena (2014).

6 En este sentido, véanse García y Rodríguez (2018) y Rodríguez (2013). 
Asimismo, otro de los objetivos específicos pasa por conseguir que el mayor número de estudiantes rellene tanto la encuesta inicial como la final. Para ello, se tratará de diseñar una encuesta sencilla, que pueda completarse y enviarse en pocos minutos, pero que contenga toda la información que como docentes necesitamos obtener para realizar los correspondientes ajustes del programa y de los materiales de nuestras asignaturas a la idiosincrasia de cada grupo, haciendo una adaptación específica no por asignatura per se, sino por grupo de clase. Además, se buscará crear una continuidad entre la encuesta inicial y la final, de forma que la segunda sirva, entre otras cosas, para valorar la adaptación de la asignatura hecha por el docente a la luz de los resultados obtenidos en la encuesta inicial durante el avance del semestre.

Ello es especialmente interesante cuando el número de horas docentes impartidas no supera el mínimo exigido por el centro educativo para confeccionar la encuesta genérica del centro, esto es, la encuesta final de la asigna-

Para no ser una rémora, la encuesta inicial ha de resultar sencilla y debe poder rellenarse en pocos minutos tura que el propio centro pone a disposición de los estudiantes al final del semestre para todas las asignaturas que cumplan ese mínimo. Esto sucede en algunos casos en asignaturas de tipo seminario, para las que la medida propuesta en este trabajo permitiría cubrir ese déficit de evaluación oficial de la universidad, proveyendo al profesorado que imparte la asignatura de unos datos tanto o más útiles que los que se hubieran generado con la encuesta-tipo y que, igualmente, podrá hacer valer en los procesos de evaluación docente ulteriores.

El éxito de todo ello depende de la configuración de la primera encuesta como una verdadera encuesta inicial y no como una encuesta de desarrollo del curso. El carácter inicial ha de asociarse a la realización en las primeras semanas del curso o, incluso, antes del inicio del mismo. Sin embargo, esta limitación temporal necesariamente conducirá a su vez a una limitación cuantitativa del volumen de personas que realizan la encuesta.

\section{Método}

El método de esta práctica docente se basa en el empleo de plataformas educativas, como puede ser Moodle. En particular, la metodología empleada por la autora de este trabajo es de tipo no experimental, ex ante y ex post facto, y de corte descriptivo. Como se ha indicado, se trata de configurar y emplear una encuesta inicial específica que los estudiantes realizarán en las primeras semanas de curso y que se verá completada con una encuesta final, siendo ambas adicionales a las encuestas generalistas que pueda proporcionar la propia universidad. Para que los objetivos perseguidos se cumplan, las preguntas y los tipos de respuestas posibles de ambas encuestas han de construirse de forma coherente. 
El éxito del empleo de la herramienta de la encuesta se basa en el anonimato, en el reducido número de ítems configurado por el docente en cada encuesta, en el poco tiempo que los estudiantes han de dedicar a su contestación y en la facilidad de recogida automática de los datos de naturaleza cuantitativa -en el mismo momento de cierre de la encuesta-, así como en la propia creación automática de estadísticas por la plataforma educativa empleada. Este último dato es fundamental a la hora de comparar la información relativa a los distintos grupos, a las diferentes asignaturas y a los diversos cursos académicos en los que se imparte docencia.

En particular, la plataforma Moodle pone a disposición del profesorado dos tipos de herramientas que pueden ser útiles en el proceso enseñanza-aprendizaje a la hora de articular los objetivos perseguidos:

- Cuestionarios.

- Encuestas.

La gran ventaja del cuestionario es la de permitir programar una retroalimentación automática que recibe el estudiante tras contestar cada pregunta. De ahí que la utilidad del cuestionario sea indudable cuando se emplea para evaluar definiciones, conceptos y relaciones entre estos, permitiendo que el docente programe con antelación una respuesta genérica a toda contestación de los estudiantes o una retroalimentación específica en función de la respuesta seleccionada por cada alumno. Sin embargo, la utilidad del cuestionario para que el estudiante valore la docencia o aporte datos e información a su docente es escasa.

De entre ambas opciones, la autora de este trabajo prefiere emplear la opción encuesta y hacer una retroalimentación aparte, subiendo a la plataforma educativa, una semana después del cierre de la encuesta, un documento PDF en el que se reflexiona sobre los resultados para que todos los estudiantes -hayan completado o no la encuesta- sean conscientes de lo que se ha contestado globalmente, como grupo, y para que estén al día de qué medidas ha decidido tomar la docente acerca de la adaptación del cronograma, así como de la metodología empleada en la articulación, en el desarrollo y en la evaluación de los seminarios que configuren esa asignatura.

\section{El docente debe reflexionar sobre los resultados de la encuesta inicial y acerca de las medidas de adaptación tomadas, dando feedback de ello a todos los estudiantes en poco tiempo}

Es fundamental que el documento sobre reflexiones sea considerado por el profesorado un compromiso de mejora y adaptación real y que se suba a la plataforma educativa con la misma publicidad que tuvo la encuesta inicial, esto es, avisando igualmente a los estudiantes de la existencia de este documento. Para que sea realmente efectivo, el documento sobre reflexiones ha de realizarse con prontitud tras la terminación del plazo de envío de la encuesta inicial. Por ello, debe tomarse en consideración el tiempo invertido por el docente en la implementación de esta herramienta y sus consecuencias adaptativas en estas primeras semanas. 
Es importante articular la herramienta de forma anónima, para promover la mayor participación del estudiantado y su sinceridad en la respuesta, al transmitir a los alumnos un mensaje menos intrusivo: para el docente lo importante es saber qué se ha dicho y no quién lo ha dicho. Adicionalmente, el citado anonimato permite cumplir con la normativa sobre protección de datos personales sin tener que solicitar a los estudiantes un consentimiento expreso y previo al inicio de la encuesta ${ }^{7}$. Ello se extiende a la posibilidad de hacer uso de los datos que obtengamos de encuestas iniciales y finales de naturaleza anónima en publicaciones científicas y congresos de innovación docente, sin necesidad de recabar un consentimiento adicional, que sí sería necesario si la herramienta no se hubiera configurado de forma que se busque el anonimato de las respuestas.

La herramienta de la encuesta permite un grado de adaptación elevado, siendo muy amplio el margen de libertad de los docentes para adaptar tanto el número de preguntas como su contenido, así como para variar la naturaleza de la pregunta, eligiendo entre respuestas de «sí» o «no», elección entre respuestas múltiples predeterminadas, texto libre para los estudiantes, etc. Además, la versatilidad de esta herramienta facilita la duplicación para su uso en cursos académicos ulteriores, permitiendo modificar, eliminar y añadir preguntas. En cualquier caso, el objetivo no pasa por hacer uso de las encuestas-tipo que ofrecen Moodle u otras plataformas educativas -encuestas COLLES (constructivist on-line learning environment survey) y ATTLS (attitudes towards thinking and learning survey)-, sino que se busca personalizar el contenido de cada encuesta para que aumente proporcionalmente la utilidad de la información que proporciona al profesorado (Castro, 2004).

A efectos de conseguir que el mayor número de estudiantes rellene tanto la encuesta inicial como la final, estas deben poder realizarse en pocos minutos y tener un reducido número de preguntas. En particular, la encuesta implementada por la autora de este trabajo para el curso académico 2018/2019 se podía realizar en menos de 4 minutos y estaba conformada por menos de 10 preguntas.

Las plataformas educativas actuales permiten que las preguntas sean confeccionadas con un amplio margen de libertad, dado que no establecen un número máximo de carac-

7 Se califica como «dato personal» cualquier información concerniente a personas físicas que, por sí misma o sumada a otras informaciones, permita identificar a la persona. En particular, el artículo 4.1 del Reglamento (UE) 2016/679 del Parlamento Europeo y del Consejo, de 27 de abril de 2016, general de protección de datos, contiene la siguiente definición:

Toda información sobre una persona física identificada o identificable («el interesado»); se considerará persona física identificable toda persona cuya identidad pueda determinarse, directa o indirectamente, en particular mediante un identificador, como, por ejemplo, un nombre, un número de identificación, datos de localización, un identificador en línea o uno o varios elementos propios de la identidad física, fisiológica, genética, psíquica, económica, cultural o social de dicha persona.

Para más información véase Cazurro (2017). 
teres de cada pregunta. En particular, la autora de este trabajo ha optado por preguntas que únicamente admiten la opción de respuesta «sí/no» con la finalidad de permitir, por un lado, un ahorro de tiempo de respuesta de los estudiantes, pero también para procurar una mayor facilidad a la hora de comparar los resultados obtenidos entre las diferentes asignaturas impartidas, sin necesidad de acudir a gráficas más complejas y sin permitir respuestas de texto abierto que, generalmente, desincentivan la contestación, por perderse, en cierta medida, el carácter totalmente anónimo de la respuesta.

Los alumnos también tienen ese margen de libertad, toda vez que la encuesta, para ser realmente libre, ha de configurarse como «no evaluable» y que este dato sea visible por los estudiantes antes de iniciar su respuesta. Además, es importante configurar el carácter opcional de cada respuesta de la encuesta de forma que el estudiante que decida realizar la encuesta no tenga por qué contestar a todas las preguntas, sino que puede dejar una, varias o todas ellas sin contestar si así lo desea. El docente verá cuántas personas contestaron la encuesta y cuántas respuestas hubo por pregunta, pudiendo deducir fácilmente el nivel de abstención de cada pregunta. Este último dato es especialmente útil de cara a poder adaptar para cursos ulteriores las preguntas que se han contestado menos, tras hacer una reflexión comparativa con el contenido y la forma de las preguntas que se han contestado más.

El proceso de redacción, así como la forma de adaptación llevada a cabo en el curso académico siguiente son acciones en las que el docente ha de ser consciente de la utilidad de invertir un periodo temporal suficiente, pues de la idiosincrasia de la encuesta en su conjunto, y, en particular, de cada una de las preguntas, se deriva la clave del éxito de esta propuesta. Si la primera vez que se emplea esta herramienta el tiempo dedicado a garantizar su éxito es menor del que hubiera sido el adecuado y los resultados conseguidos en las encuestas inicial y, sobre todo, en la encuesta final no son los deseados, puede ser probable que el docente no encuentre utilidad ni motivación suficientes para mejorar esta herramienta en cursos posteriores y/o para su utilización como evidencia en los procesos de acreditación y de evaluación docente.

\section{Diseño y confección}

La autora de este trabajo implementó la herramienta propuesta en todas sus asignaturas del primer semestre del curso 2018/2019, empleando la plataforma educativa Moodle. Se tomará como ejemplo, por un lado, la asignatura Seminarios de Derecho de Obligaciones y Contratos, que es una asignatura de carácter obligatorio impartida en el primer semestre del segundo curso del grado de Derecho y que consta de 9 créditos ECTS (european credit transfer system). Por otro lado, se expondrán de manera comparativa los resultados obte- 
nidos en la asignatura Seminarios de Derecho de la Persona, materia obligatoria del primer semestre del primer curso del grado de Derecho (3 créditos ECTS).

Empezaremos la reflexión con la encuesta realizada en Seminarios de Derecho de Obligaciones y Contratos. Se trata de una asignatura con 35 matriculados. De ellos, 14 estudiantes contestaron la encuesta, lo que supone un $40 \%$ de las personas matriculadas. La docencia comenzó el 8 de octubre de 2018 y la encuesta permaneció abierta entre el 1 y el 14 de octubre de 2018. Los estudiantes fueron avisados en dos ocasiones de la existencia de la encuesta inicial mediante mensajes de Moodle.

La encuesta inicial constaba de un total de seis preguntas, cuyas respuestas podían ser «sí/no», permitiendo la opción de no contestar a una o a varias de las preguntas. En particular, las preguntas fueron las siguientes:

1. ¿Quiere que los seminarios se basen en casos reales, adaptados a la duración de las clases, en los que poder actuar como abogados/as?

2. ¿Quiere conocer los proyectos de mejora y modernización de algunas de las materias basilares contenidas en el Código Civil y quiere analizar cómo esas propuestas se adaptan a la realidad social actual?

3. ¿Está familiarizado/a con el trabajo en equipo?

4. ¿Le gustaría combinar, en la resolución de los seminarios, el trabajo individual y el trabajo en equipo?

5. ¿Está familiarizado/a con la exposición oral de los resultados de sus estudios previos?

6. ¿Le gustaría mejorar las técnicas de expresión escrita y oral como jurista?

El 19 de octubre de 2018 -5 días después de finalizar el plazo dado para contestar la encuesta- la profesora subió a la plataforma educativa el documento «Reflexiones sobre los resultados de la encuesta inicial y medidas de adaptación», lo que se corresponde con la semana en la que los estudiantes realizaron el segundo seminario no evaluable. En esta asignatura se hace un seminario cada semana. Una semana es evaluable y otra no. Los seminarios se dividen por bloques temáticos y cada bloque temático suele constar de dos seminarios -en algunos casos de tres-. Así, por ejemplo, el primer y el segundo seminario de la asignatura se referían a la cuestión «Los sujetos de la relación obligatoria. Las obligaciones sometidas a condición». Al tratarse de una asignatura construida mediante la metodología del aprendizaje basado en proyectos, el segundo seminario siempre parte del mismo supuesto de hecho que el primero, y el caso completo se va construyendo a medida que se avanza en las tareas, pudiendo ser conscientes los estudiantes del lugar del que partían en el primer seminario y del sitio al que han llegado al terminar la última tarea del segundo seminario, esto es, siendo conscientes del desarrollo y terminación del proyecto. En esa fecha, los estudiantes habían realizado un único 
seminario evaluable para el que fue imposible introducir medidas de adaptación a los resultados de la encuesta, pues los materiales y los documentos explicativos de la tarea que había que realizar, la forma de desarrollo del seminario y su evaluación, incluida su rúbrica, habían sido subidos a la plataforma educativa por la profesora el 1 de octubre de 2018 para cumplir con el calendario académico.

Por su parte, en la asignatura Seminarios de Derecho de la Persona, la participación en la encuesta inicial fue mayor, ya que 22 de los 37 estudiantes matriculados en la asignatura rellenaron la encuesta. La publicidad dada a la existencia de la encuesta y las características de la misma eran equivalentes a las de la asignatura Seminarios de Derecho de Obligaciones y Contratos.

\section{Resultados y adaptaciones}

Los resultados de la encuesta inicial de la asignatura Seminarios de Derecho de Obligaciones y Contratos se detallan en la figura siguiente:

Figura 1. Encuesta inicial de la asignatura Seminarios de Derecho de Obligaciones y Contratos

1

¿Quiere que los seminarios se basen en casos reales, adaptados a la duración de las clases, en los que poder actuar como abogados/as?

\begin{tabular}{|c|c|c|}
\hline Respuesta & Media & Total \\
\hline Sí & $93 \%$ & 13 \\
\hline No & $7 \%$ & 1 \\
\hline
\end{tabular}

2 ¿Quiere conocer los proyectos de mejora y modernización de alguna de las materias basilares contenidas en el Código Civil y quiere analizar cómo esas propuestas se adaptan a la realidad social actual?

\begin{tabular}{lrr} 
Respuesta & Media & Total \\
\hline Sí & $100 \%$ & 12 \\
No & & 0 \\
\hline Total & $86 \%$ & $12 / 14$
\end{tabular}

3 ¿Está familiarizado/a con el trabajo en equipo?

\begin{tabular}{lrr} 
Respuesta & Media & Total \\
\hline Sí & $100 \%$ & 14 \\
No & $100 \%$ & 0 \\
\hline Total & $14 / 14$
\end{tabular}


¿Le gustaría combinar en la resolución de los seminarios el trabajo individual y el trabajo en equipo?

\begin{tabular}{|c|c|c|}
\hline Respuesta & Media & Total \\
\hline Sí & $93 \%$ & 13 \\
\hline No & $7 \%$ & 1 \\
\hline
\end{tabular}

5 ¿Está familiarizado/a con la exposición oral de los resultados de sus estudios previos?

\begin{tabular}{|c|c|c|}
\hline Respuesta & Media & Total \\
\hline Sí & $71 \%$ & 10 \\
\hline No & $29 \%$ & 4 \\
\hline Total & $100 \%$ & $14 / 14$ \\
\hline
\end{tabular}

$6 \quad$ ¿Le gustaría mejorar las técnicas de expresión escrita y oral como jurista?

\begin{tabular}{|c|c|c|}
\hline Respuesta & Media & Total \\
\hline Sí & $92 \%$ & 12 \\
\hline No & $8 \%$ & 1 \\
\hline Total & $93 \%$ & $13 / 14$ \\
\hline
\end{tabular}

Las adaptaciones realizadas por la profesora fueron las siguientes:

- A la luz de la respuesta dada a la primera pregunta, puede afirmarse que un estudiante de segundo curso del grado de Derecho ya es consciente del rol que va a desempeñar en la sociedad, como abogado del futuro, y de que quiere empezar a desarrollarlo ya. Es por ello que todos los seminarios restantes fueron articulados mediante la presentación de un caso real, si bien adaptado por la profesora para eliminar referencias a cuestiones paralelas no centradas en la concreta temática que se iba a estudiar en ese seminario.

En cada seminario, la tarea consiste en ponerse en el papel de abogados que han de emitir un informe jurídico breve, redactar un contrato en el que se han introducido previamente errores voluntarios por la profesora o asesorar de forma informal a su cliente acerca de la procedencia o no de las ideas de actuación que este último pueda tener. En muchos casos, los nombres de las partes contractuales coincidían entre seminarios para que los estudiantes interiorizaran mejor la evolución de los ciudadanos -clientes- a los que han de asesorar o defender en cada seminario.

- El hecho de que el $100 \%$ de las personas que contestaran a la segunda pregunta lo hicieran afirmativamente condujo a la docente a introducir en todos los semi- 
narios una cuestión relativa a los proyectos de reforma de la normativa vigente. En particular, se han empleado la Propuesta de Código Civil de la Asociación de Profesores de Derecho Civil, presentada en 2018, y la Propuesta de modernización del Código Civil en materia de obligaciones y contratos de 2009, elaborada por la Comisión General de Codificación.

- Especialmente útil para la profesora fue el hecho de que el $100 \%$ de las personas que contestaron a la tercera pregunta afirmaran estar familiarizadas con el trabajo en equipo, así como el dato dimanado de la respuesta a la cuarta pregunta: el $93 \%$ preferían combinar la resolución de tareas en equipo con el trabajo individual. Como adaptación, la profesora hizo una combinación de ambas formas de trabajo: introduciendo en todos los seminarios una primera fase individual de trabajo y una segunda etapa en equipo. El $60 \%$ de seminarios evaluables implicaban tareas que había que realizar en equipo, mientras que el $40 \%$ restante eran evaluados de manera individual. Ello conduce a un mayor dinamismo del seminario y puede ser útil para aumentar la atención de los estudiantes. En la citada segunda fase grupal, la profesora dividía la clase en comités de expertos que podían optar por mantenerse como equipos estables para el resto de seminarios o modificar la composición del equipo -previa comunicación con la profesora- y que debían configurar una única respuesta de grupo a cada tarea, partiendo del debate acerca de la respuesta individual previamente dada.

- La respuesta afirmativa a la quinta pregunta fue más baja, en comparación con las anteriores: el $29 \%$ de las personas que respondieron a esta pregunta declararon que no estaban familiarizadas con la exposición oral de los resultados tras un trabajo previo. Sin embargo, como complemento a lo anterior, en el caso de la sexta pregunta, el $92 \%$ de las personas que contestaron indicaron su deseo de mejorar la expresión oral y escrita como juristas. Como medida de adaptación, la profesora optó por reservar 15 minutos de cada clase, dentro de la segunda fase grupal de cada seminario, a la exposición oral de los resultados, debiendo nombrar cada grupo de trabajo a un único ponente por cada día y debiendo variar la identidad de ese ponente cada semana con el objetivo de que todos los estudiantes expusieran oralmente al menos una vez a lo largo del semestre.

En el caso de la asignatura Seminarios de Derecho de la Persona, que no se imparte en segundo, sino en el primer curso del grado de Derecho, y que es igualmente obligatoria, la participación en la encuesta inicial, como se ha indicado, fue mayor y la comparación con las respuestas dadas nos ha permitido inferir algunas diferencias entre estudiantes de primer y de segundo curso que seguidamente se expondrán. Los resultados se muestran en la figura 2. 
Figura 2. Encuesta inicial de la asignatura Seminarios de Derecho de la Persona

1

¿Quiere que los seminarios se basen en casos reales, adaptados a la duración de las clases, en los que poder actuar como abogados/as?

\begin{tabular}{lrr} 
Respuesta & Media & Total \\
\hline Sí & $100 \%$ & 21 \\
No & & 0 \\
\hline Total & $95 \%$ & $21 / 22$
\end{tabular}

2 ¿Quiere conocer los proyectos de mejora y modernización de alguna de las materias basilares contenidas en el Código Civil y quiere analizar cómo esas propuestas se adaptan a la realidad social actual?

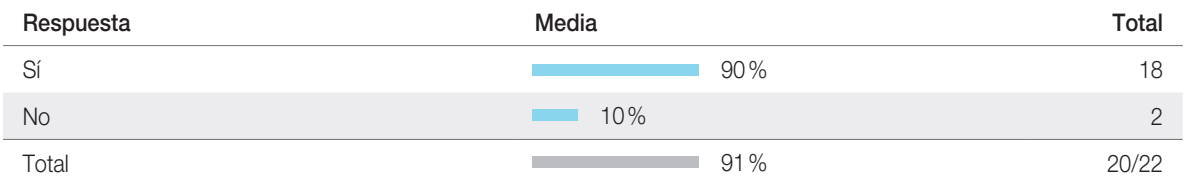

3 ¿Está familiarizado/a con el trabajo en equipo?

\begin{tabular}{lrr} 
Respuesta & Media & Total \\
\hline Sí & $85 \%$ & 17 \\
No & $15 \%$ & 3 \\
\hline Total & & $91 \%$
\end{tabular}

4 ¿Le gustaría combinar en la resolución de los seminarios el trabajo individual y el trabajo en equipo?

\begin{tabular}{lrr} 
Respuesta & Media & Total \\
\hline Sí & $100 \%$ & 19 \\
No & & 0 \\
\hline Total & $86 \%$ & $19 / 22$
\end{tabular}

5 ¿Está familiarizado/a con la exposición oral de los resultados de sus estudios previos?

\begin{tabular}{lrr} 
Respuesta & Media & Total \\
\hline Sí & $27 \%$ & 4 \\
No & $73 \%$ & 11 \\
\hline Total & $68 \%$ & $15 / 22$
\end{tabular}

$6 \quad$ ¿Le gustaría mejorar las técnicas de expresión escrita y oral como jurista?

\begin{tabular}{lrr} 
Respuesta & Media & Total \\
\hline Sí & $100 \%$ & 20 \\
No & & 0 \\
\hline Total & $91 \%$ & $20 / 22$
\end{tabular}


Los estudiantes de la asignatura Seminarios de Derecho de la Persona demostraron estar menos familiarizados con el trabajo en equipo y poco familiarizados con la exposición oral de los resultados de sus trabajos previos, pero afirmaron tener un interés mayor en cumplir el rol de abogados en la resolución de tareas y en mejorar sus técnicas de exposición oral y escrita. Como medida de adaptación incluida por la profesora, distinta de las ya expuestas, ha de destacarse el mayor tiempo dedicado a la exposición oral de las conclusiones de los trabajos grupales, equivalente a 30 minutos de cada seminario (siendo la duración del seminario de 1 h 30 min). Además, la profesora destinó un tiempo menor a la explicación de las propuestas de modernización o mejora de la normativa vigente, siendo consciente de que los alumnos del primer curso del grado de Derecho tienen un interés menor en la comparativa crítica entre el ordenamiento actual y las reformas futuras, pues esta competencia será trabajada con mayor intensidad en cursos ulteriores.

En lo que al análisis de la encuesta final de curso se refiere, únicamente la asignatura Seminarios de Derecho de Obligaciones y Contratos tuvo encuesta oficial. No así la asignatura Seminarios de Derecho de la Persona, al ser el número de horas de docencia efectiva impartidas por la profesora inferior a 10, por lo que los datos aquí expuestos se basan en la encuesta específica preparada por la profesora de forma anónima en Moodle en esta segunda asignatura; circunstancia que, como se ha indicado, multiplica la utilidad de la herramienta de la encuesta personalizada y permite a los docentes garantizar la prueba empírica de la llevanza de un proceso de adaptación personalizada para el grupo y el seguimiento de su estudiantado. Esta encuesta final específica servirá también de prueba o evidencia en los procesos de acreditación y evaluación docente.

De las citadas respuestas se puede inferir el cumplimiento del citado objetivo de crear en los estudiantes la consciencia acerca del esfuerzo realizado por la docente para adaptar los seminarios a las expectativas de aprendizaje y a ese perfil del estudiantado, además de crear o aumentar en cada estudiante la consciencia acerca del propio proceso de aprendizaje y del seguimiento de su docente. Tal y como se muestra en la figura 3, las respuestas afirmativas fueron del $100 \%$ en las preguntas acerca del interés y del carácter actual de los materiales empleados, así como en las preguntas sobre la utilidad del trabajo en equipo a la hora de fijar conocimientos y sobre el aumento del interés por la materia a lo largo del semestre ${ }^{8}$. Por su parte, las preguntas acerca del efecto positivo del trabajo en equipo para la mejora de la redacción jurídica y sobre la utilidad de la exposición oral para el mejor entendimiento de la asignatura se contestaron de forma afirmativa en el $95 \%$ de los casos. Un dato llamativo es que, a diferencia de lo que sucedía en la encuesta inicial, en el caso de las encuestas finales los estudiantes que deciden realizar y enviar la encuesta contestan a todas las preguntas, sin abstenerse en ninguna de ellas; dato que puede deberse a la propia evolución del grado de consciencia del estudiantado acerca de la relevancia de las encuestas para el personal docente.

8 Para evitar duplicidades, el texto de las preguntas que componen la encuesta final no se reproduce en el cuerpo de este trabajo, sino que se refleja únicamente en la figura 3. 
Figura 3. Encuesta final de la asignatura Seminarios de Derecho de la Persona

1 ¿A lo largo del semestre ha aumentado su interés por la materia objeto de estudio en la asignatura?

\begin{tabular}{lrr} 
Respuesta & Media & Total \\
\hline Sí & $100 \%$ & 20 \\
No & $100 \%$ & 0 \\
\hline Total & $10 \%$
\end{tabular}

2 ¿El trabajo en equipo le ha ayudado a fijar conocimientos?

\begin{tabular}{lrr} 
Respuesta & Media & Total \\
\hline Sí & $100 \%$ & 20 \\
No & $100 \%$ & 0 \\
\hline Total & $100 / 20$
\end{tabular}

3 ¿El trabajo en equipo le ha ayudado a mejorar su redacción jurídica?

\begin{tabular}{|c|c|c|}
\hline Respuesta & Media & Total \\
\hline Sí & $95 \%$ & 19 \\
\hline No & $5 \%$ & 1 \\
\hline
\end{tabular}

$4 \quad$ ¿La exposición oral en clase ha favorecido el entendimiento de la asignatura?

\begin{tabular}{|c|c|c|}
\hline Respuesta & Media & Total \\
\hline Sí & $95 \%$ & 19 \\
\hline No & $5 \%$ & 1 \\
\hline
\end{tabular}

$5 \quad$ ¿Los materiales empleados en la asignatura le han resultado interesantes y actuales?

\begin{tabular}{lrr} 
Respuesta & Media & Total \\
\hline Sí & $100 \%$ & 20 \\
No & $100 \%$ & 0 \\
\hline Total & $20 / 20$
\end{tabular}

Por su parte, en la encuesta genérica de final de curso llevada a cabo por la universidad para la asignatura Seminarios de Derecho de Obligaciones y Contratos, en el apartado «Comentarios Abiertos», se ve reflejada la citada consciencia de los estudiantes acerca de la implicación buscada por la docente. En este sentido pueden interpretarse las siguientes afirmaciones anónimas: «Muy práctico todo, además de la importancia que da a sus alumnos. Se aprende mucho», "Acercarse a los estudiantes para crearles un interés real es algo tan difícil como satisfactorio», «La buena organización de la asignatura y el buen ritmo de trabajo semanal, que motiva mucho a venir a clase», «La manera de abordar los seminarios, 
haciendo grupos y luego exponiendo nuestras preguntas, hacen las clases dinámicas, amenas e interesantes. Las pruebas de evaluación continua son buenas y asequibles. Tal y como están diseñadas, se aprende mucho» ${ }^{9}$.

El siguiente paso que se va a implementar en la asignatura Seminarios de Derecho de Obligaciones y Contratos en el próximo curso académico es la realización de una encuesta final personalizada mediante Moodle, como se ha hecho con la asignatura Seminarios de Derecho de la Persona, con la que se pueda contrastar si el impacto positivo de la adaptación realiza-

De las respuestas a la encuesta final se infiere la consciencia de los estudiantes sobre el esfuerzo del profesor en la labor de adaptación de la docencia tras la encuesta inicial da gracias a la información suministrada en la encuesta inicial por los estudiantes fue el mismo o distinto que el de la asignatura Seminarios de Derecho de la Persona. Con ello se tratará de estudiar si la herramienta de la encuesta inicial y el compromiso de adaptación de materiales y técnicas dimanado de ella es valorada de la misma manera por estudiantes de distintos cursos del grado de Derecho.

\section{Conclusiones}

Los resultados obtenidos con la implementación de la propuesta aquí expuesta señalan que el uso de esta herramienta es considerado beneficioso dentro del proceso enseñanza-aprendizaje. Los estudiantes confieren un valor positivo a la herramienta de la encuesta inicial y, sobre todo, al compromiso de adaptación dimanado de ella, a cumplir por el docente durante ese semestre. Todo ello permite su mayor implicación en la asignatura y reforzar la relación profesorado-estudiantado. Para el docente resulta gratificante ver que sus estudiantes analizan la evolución de su docencia y valoran positivamente su esfuerzo, sobre todo cuando el nivel de dedicación ha sido elevado. Además, la herramienta de la encuesta permite a los docentes conocer de primera mano el mantenimiento, el incremento o la reducción de la implicación del grupo.

Este último factor merece ser destacado, pues resulta complicado de analizar si la encuesta es de tipo genérico, es decir, si es creada de forma global por el centro educativo y no personalizada por cada docente.

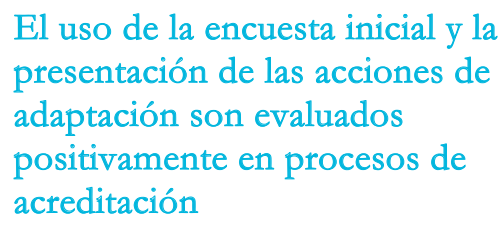

9 Las evidencias enviadas por la universidad a la docente no se incluyen en este estudio, pues los comentarios abiertos de los estudiantes se muestran junto con las gráficas de desempeño general de la profesora-autora de este trabajo. Dado que estas tablas de valoración general no son evidencia de la idoneidad de la encuesta inicial de curso, la autora ha preferido omitir su reproducción en este trabajo. 
Dicho trabajo será asimismo valorado en sede de acreditaciones y en procesos de evaluación docente, como es el programa Docentia, donde se da un peso importante al uso de encuestas en los diferentes tramos del aprendizaje. El modelo propuesto permite a cada docente hacerse con evidencias y datos de naturaleza objetiva que serán muy útiles para su empleo en el siguiente programa de Docentia en el que pretenda participar. Este reconocimiento garantizará la continuidad de las acciones emprendidas, sirviendo este modelo -si bien sujeto a actualización y mejora continuada- de referencia para cursos académicos posteriores. No se puede olvidar que uno de los objetivos del programa Docentia es el diseño y acceso a actividades de formación del profesorado. De ahí que el uso extensivo de la herramienta de la encuesta inicial pueda servir a las universidades para tomar conciencia de la importancia de crear programas de formación docente que sirvan para la mejora en la confección de encuestas personalizadas para cada docente -a implantar al inicio de su docencia-, adicionales a las encuestas generalistas que la propia universidad ofrezca al término del semestre. Además, el modelo propuesto proporciona a los docentes un grado de autonomía e independencia notables, que sirve asimismo de espacio para el ensayo y para la mejora semestral de un modelo de prueba, error y adaptación.

Con todo, el concreto impacto en la mejora docente y el efecto positivo que el profesorado puede obtener de ello deben continuar siendo estudiados. En particular, habrá que dedicar más tiempo a la tarea de adaptación de las formas de evaluación entre los docentes que imparten las asignaturas de seminarios y magistrales para que el uso de las encuestas inicial y final personalizadas en seminarios tenga igualmente un reflejo en las clases magistrales y se pueda comprobar si ello repercute o no y de qué forma en la percepción global de los estudiantes y en su rendimiento no solo en seminarios, sino también en las clases de tipo magistral, y, con ello, en la evaluación final de la asignatura. Si ello se traduce en una mejora global de los resultados académicos, seguramente lleve consigo una valoración general muy positiva por el estudiantado y por la universidad.

Haber llevado a cabo esta práctica docente en el primer semestre del curso 2018/2019 ha permitido a la autora de este estudio experimentar un grado de frustración menor en las fases de diseño, ejecución y evaluación de tareas al conocer mejor el perfil de los estudiantes a los que se dirige la docencia y tener un tiempo de adaptación de la materia impartida teniendo en cuenta los resultados de la encuesta inicial. Se desconoce si ello se ha traducido igualmente en una disminución de la frustración propia del estudiantado en las diferentes fases de evaluación. Por todo ello, puede afirmarse que la herramienta en cuestión ha cumplido el principal objetivo perseguido, pudiendo beneficiarse en ulteriores cursos y en posteriores procesos de acreditación y evaluación docente de los resultados alcanzados, si bien el impacto en la mejora docente habrá de seguir siendo analizado.

La encuesta inicial puede adaptarse a cursos ulteriores, mejorando las preguntas y el tipo de respuestas 


\section{Referencias bibliográficas}

Agencia Nacional de Evaluación de la Calidad y Acreditación. (2015). Programa Docentia. Programa de apoyo para la evaluación de la actividad docente del profesorado universitario. Integración y actualización de la documentación del programa 2015. V1. Recuperado de <http://www.aneca.es/ Programas-de-evaluacion/Evaluacion-insti tucional/DOCENTIA/Documentacion-delprograma> (consultado en junio de 2019).

Castro López-Tarruella, E. (2004). Moodle: manual del profesor. Recuperado de $<$ https://moodle.org/file.php/11/manual_ del_profesor/Manual-profesor.pdf> (consultado en junio de 2019).

Cazurro Barahona, V.(2017). Las universidades públicas y el coste de la garantía del derecho a la protección de datos personales. Actualidad Administrativa, 4.

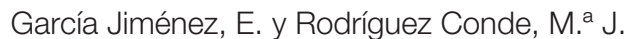
(2018). El programa Docentia: fundamentos, desarrollo e implicaciones. Revista de Educación y Derecho, 17, 1-26.
Iglesias Rodríguez, A., Olmos Migueláñez, S., Torrecilla Sánchez, E. M. ${ }^{a}$ y Mena Marco, J. J. (2014). Evaluar para optimizar el uso de la plataforma Moodle (studium) en el departamento de didáctica, organización y métodos de investigación. Tendencias Pedagógicas, 23, 155-170.

Marsh, H. W. (1987). Students' evaluations of university teaching: research findings, methodological issues, and directions for future research. International Journal of Educational Research, 11(3), 253-388.

Pablo González, G. de. (2017). Factores que favorecen la presencia docente en entornos virtuales de aprendizaje. Tendencias Pedagógicas, 29, 43-58.

Rodríguez Conde, M. ${ }^{a}$ J. (1994). El autoinforme en la evaluación docente del profesorado universitario (Tesis). Salamanca: Universidad de Salamanca.

Rodríguez Espinar, S. (2013). Panorama internacional de la evaluación de la calidad en la educación superior. Madrid: Síntesis.

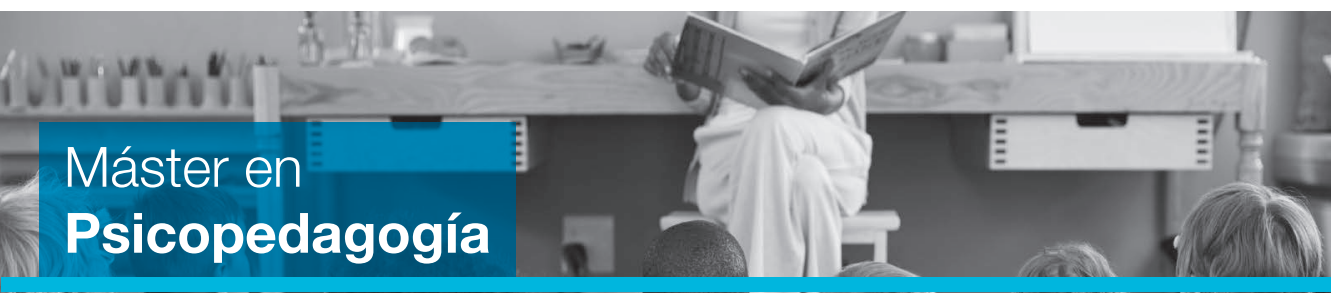

Este máster oficial [60 créditos ECTS] tiene una duración normal de 12 meses.

Dirigido a: Personas vinculadas con el mundo de la educación formal y no formal que deseen actualizar sus conocimientos. El estudiante de este máster ha de estar interesado por la labor del educador en un enfoque educativo inclusivo en el ámbito de la educación formal, y en el trabajo con diferentes grupos o colectivos sociales, favoreciendo la mejora de sus condiciones de vida y la disminución de las desigualdades por motivos de carácter social y cultural.

Objetivos: Permite el desempeño de una labor profesional especializada, avanzada y focalizada en el análisis, la planificación y la intervención para la mejora de los contextos educativos, sociolaborales y sociocomunitarios, de ahí la necesidad de una formación de posgrado que permita el desarrollo de las competencias específicas y multidisciplinares requeridas para su práctica profesional. Pretende dar cobertura a las funciones básicas de los psicopedagogos en distintos ámbitos. 


\section{Ingeniería de Tecnologías y Servicios de Telecomunicación}

El papel estratégico del sector de las TIC, y la aplicación creciente de estas en los distintos sectores de la sociedad, ha hecho aumentar la necesidad de profesionales de la telecomunicación, una demanda que crecerá exponencialmente en los próximos años. Este grado habilita para el ejercicio de la profesión de ingeniero técnico de telecomunicación, otorgando las competencias necesarias para conseguir las atribuciones profesionales de un ingeniero técnico de telecomunicación y ofreciendo una formación que capacita al estudiante a la hora de analizar, diseñar, implementar, explotar y gestionar sistemas, componentes y procesos del ámbito de las TIC.

\section{Psicología (Rama CC. de la Salud)}

Siguiendo el modelo científico-profesional de psicólogo (o scientist-practitioner), se trata de aportar a los alumnos los conocimientos científicos necesarios para comprender, interpretar, analizar y explicar el comportamiento humano, así como para evaluar e intervenir en el ámbito individual y social, con el fin de que los psicólogos y la psicología promuevan y mejoren la salud y la calidad de vida de las personas.

\section{Historia}

Se conjugan los conocimientos humanísticos básicos y generalistas con el aprendizaje de las herramientas y técnicas de las nuevas TIC. Los estudiantes adquirirán la formación, los conocimientos y las habilidades necesarias para permitirles el pleno desarrollo de las funciones relacionadas con la investigación y la enseñanza de la historia, con la finalidad de que comprendan y hagan comprensibles a los demás los acontecimientos del pasado.

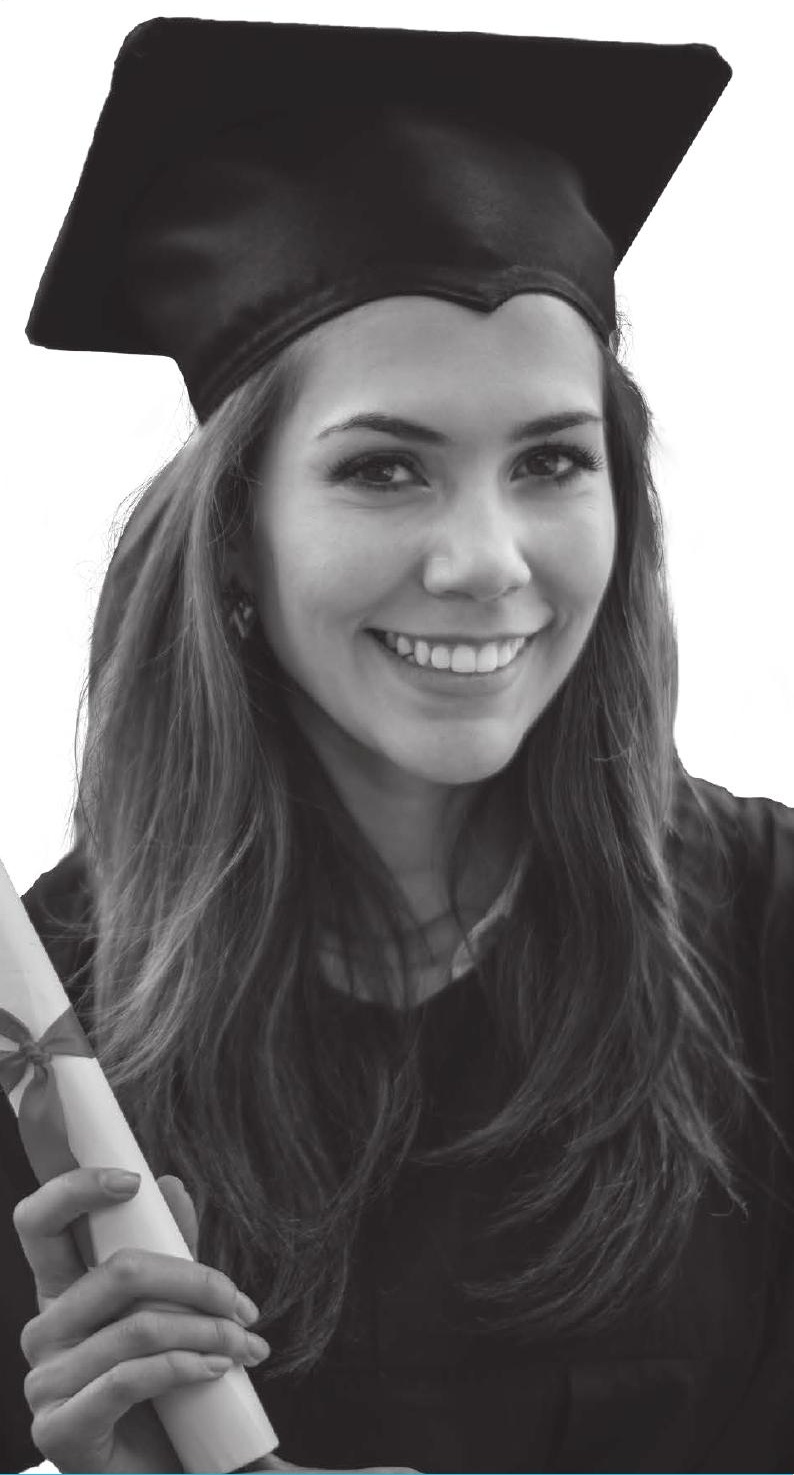

